\title{
The relation between cerebral dominance and visual analytic skills in Iraqi medical students, a cross sectional analysis
}

\author{
Nawfal Al-Hadithi', Ahmed Al-Imam², Manolia Irfan ${ }^{3}$, Mohammed Khalaf ${ }^{3}$, Sara Al-Khafaji ${ }^{3}$ \\ ${ }^{1}$ Professor, Department of Anatomy and Cell Biology, Faculty of Medicine, University of Baghdad, Iraq, ${ }^{2}$ Doctoral \\ Students, Novel Psychoactive Substances Research Unit, Department of Postgraduate Medicine, School of Life and \\ Medical Sciences, University of Hertfordshire, UK, ${ }^{3}$ Medical Student, Faculty of Medicine, University of Baghdad, Iraq
}

Background: Cerebral dominance, also known as lateralization of brain functions, is the neurologic phenomenon in which one hemisphere is dominant over the other, and will thereby exercise greater influence over certain functions. The cerebral lateralization of many vertebrates, indicates an evolutionary advantage associated with the cerebral specialization. In humans, the majority of people are of left cerebral dominance. True bilateral cerebral dominance, manifested in ambidexterity, is exceedingly rare. Aims and Objectives: To derive a statistical inference between determined parameters of cerebral dominance and the 3-dimensional visual analytic skills. The parameters are: handedness, gender, and language skills. Materials and Methods: This survey targeted an audience of undergraduate medical students, 103 of them replied and fully completed an electronic-distributed survey. All participants were blinded to the outcome and aims of the study. The survey was created via Google Forms, and made available online for three days, on the University intranet system. Compiled data resulted in 523 pages. Statistical analyses were conducted using the Statistical Package for Social Sciences, Shodor-Interactivate software, and Microsoft Excel 2016. References of this paper, were retrieved via a detailed systematic review of literature databases, while carrying out critical evaluation of evidence. The databases included: PubMed, The Cochrane Library, Scopus, OpenGrey, and Google Scholar. Results: Males and females participated in the survey (34 and 69 participants respectively), 91 of participants $(88.4 \%)$ were right-handed. Mapped participants' demographics included: age, gender, handedness, siblings' and parents' handedness, number of siblings and birth order, and number of spoken languages. The 3D-visual analytic skills, were evaluated via a cross sectional survey and objectively-evaluated via a professional visual IQ testing. Statistics proven that there was no significant correlation between the 3D-visual skills and any of the tested parameters. Significance was considered at a p-value of 0.05 or less. Conclusion: In this sample of 103 tested subjects, all of which were not professional Anatomists. Our tested hypothesis of the presence of potential correlation between handedness (and other parameters of cerebral dominance) with the three-dimensional visual analytic skills, were proven statistically to be non-existent.

Key words: Dominance, Cerebral, Laterality, Visual, Language, Dexterity, Handedness, Magnetic resonance imaging, Students, Iraq

\section{INTRODUCTION}

Cerebral dominance refers to the dominance of one cerebral hemisphere over the other in the control of
Access this article online

Website:

http://nepjol.info/index.php/AJMS DOI: 10.3126/ajms.v7i6.15205

E-ISSN: 2091-0576

P-ISSN: 2467-9100 
are right-handed, and in the vast majority of these individuals, the left hemisphere controls language-related functions. In left-handed individuals, however, only about $75 \%$ have language functions predominantly controlled by the left hemisphere. ${ }^{1,2}$ Non-dominant hemisphere plays an important role in musical understanding, composition and learning, perception of spatial relations, perception of visual and other esthetical patterns, understanding of connotations in verbal speeches, perception of voice intonation, identification of other's emotions and mood, and body language. ${ }^{3}$

A very small percentage of people are ambidextrous, having no preference for performing tasks with either hand. Ambidexterity, as in the unique case of Leonardo da Vinci (an Italian polymath). Ambidexterity occurs when a person has approximately equal set of skills with both hands and/or both sides of the body. However, true ambidexterity is extremely rare, because a person still shows preference to use a side of the body more than the other.,

From a developmental neurobiology perspective, the lateralization of brain, initializes in reference to the median longitudinal fissure, which separates the human brain into two distinct cerebral hemispheres connected by the corpus callosum. Each human's brain develops gradually and differently leading to unique cerebral lateralization in an individual. In some aspects, the hemispheres are asymmetrical; one side is slightly bigger. There are higher levels of the neurotransmitter norepinephrine on the right and higher levels of dopamine on the left. There is also more white matter on right and more grey matter on the left. Therefore, although the macrostructure of the two hemispheres appear to be almost identical, different composition of neuronal networks allow for specialized function that is different in each hemisphere. ${ }^{6-8}$

The best example of an established lateralization is that of Broca's and Wernicke's Areas (language centers) where both are often found exclusively on the left hemisphere. These areas frequently correspond to handedness. The localization of these areas, is regularly found on the hemisphere opposite to the dominant hand. Function lateralization such as semantics, prosodic, intonation, accentuation, prosody, etc. has since been called into question and largely been found to have a neuronal basis in both hemispheres. Perceptual information is processed in both hemispheres, but it is laterally partitioned: information from each side of the body is sent to the opposite hemisphere. However, visual information is partitioned somewhat differently, but still lateralized. Similarly, motor control signals sent out to the body also come from the hemisphere on the opposite side. Thus, hand preference, i.e. the dominant hand, is also related to hemisphere lateralization. ${ }^{6,9,10}$
To summarize, Linear reasoning functions of language such as grammar and word production are often lateralized to the left hemisphere of the brain. In contrast, holistic reasoning functions of language such as intonation and emphasis are often lateralized to the right hemisphere of the brain. Other integrative functions such as intuitive or heuristic arithmetic, binaural sound localization, etc. seem to be more bilaterally controlled. ${ }^{11}$

\section{MATERIALS AND METHODS}

The data presented in this study, was derived from a survey directed towards $1^{\text {st }}$ year undergraduate medical students at the largest medical university in Iraq. A survey, cross sectional, was created online using Google Forms. ${ }^{12,13}$ All the participant students have successfully completed the lower limb module of the Human Anatomy course, and were competent in identifying sectional images of Magnetic Resonance Imaging (MRI), including cross sections.

This study is considered to be of a randomized singleblinded design, in which participants were randomly selected to participate in the survey, and they were blinded towards the survey's nature and its aim. Those who delivered and distributed the survey, co-authors of this paper, were knowledgeable of the nature, structure, and objectives of the survey. The resultant data from the survey summed up to 523 pages, upon which this study was based. Each participant in the survey was allowed to take the survey only once. The study was conducted in accordance with the Ethical approval no. $620-73$, on the $15^{\text {th }}$ of May 2016. The approval is under the authority of theInstitute Review Board (IRB) and its Ethical Committee.

The survey itself, included four cross-sectional MRI images of the lower limb, which were taken from the Atlas of Human Anatomy on MRI. ${ }^{14}$ Sections were selected by professional Anatomists at three main levels: leg (two sections), knee (one section), and thigh (one section). All these sections can be normally visualized (imagined), by a non-professional Anatomist (students in this case) either from above (a superior view) or below (an inferior view). All images were fully labelled with Anatomical landmarks, and the students were only asked about how would they $1^{\text {st }}$ visually imagine the sections, either fromabove or from below.

The survey was electronically delivered via a secured university intranet system..$^{15}$ The targeted audience, was of 297 undergraduate medical students (year-1). The survey participants were oriented about the survey a week in advance, and the survey was later posted online for three successive days from the $30^{\text {th }}$ of April, 2016 to $3^{\text {rd }}$ of May, 
2016, after which, it was locked and no other submission was allowed. The response-time curve (Figure 1), showed that the majority of participants took the survey in day-1.

The parameters of Cerebral dominance, which were being correlated against how each participant visualized the MRI images, include: handedness (i.e. dominant hand), number of spoken and written languages, and subjective (self-assessment) of the 3D-visual analytic skills. The 3D-imaginative abilities were further objectively confirmed by using a specialized 3D-visual IQ testing delivered online via a professional IQ testing society, the International HighIQ Society. ${ }^{16}$ Demographic data (Figure 2) included: age, gender, number of siblings, birth order (rank), in addition to parents' and siblings' handedness.

Additionally, a systematic literature review, was conducted from the $1^{\text {st }}$ of April 2016 to the $10^{\text {th }}$ of June 2016, concerning the concept of cerebral dominance and laterality, and previous studies and surveys on the topic. Specific and pre-determined keywords (including $\mathrm{MeSH}$ keywords), were applied across medical and paramedical databases: PubMed, The Cochrane Library, Scopus, Open Grey, and Google Scholar. This search methodology, was aiming to extract the most relevant and up-to-date literature material, which were published in professional and highimpact journals in the past 5 years (2012-2016). However, other literature materials and papers, were also considered when found relevant and of high level-of-evidence.

Total number of papers that were initially mapped were 32 . The papers and textbooks, were later filtered by reading: the paper title, abstract, and full text. This was followed by critical analysis using critical appraisal tools suitable for each type of retrieved literature papers. Duplicate papers were excluded. Accordingly, only twenty-one reference materials were finally used in the citation of this paper.

Statistical analyses of this paper, were conducted using the Statistical Package for Social Sciences (SPSS version 20.0), Shodor-Interactivate software. ${ }^{17}$ Other forms of data analysis and visual presentations, were done in Microsoft Excel 2016. Further, data were numerically encoded, to perform specialized statistical analysis with SPSS, these were: Cbisquare and Pearson's correlation test. Significance is considered when $\mathrm{p}$ value is less than 0.05

\section{RESULTS AND DISCUSSION}

The survey was successfully and fully completed by 103 medical students $(34.68 \%$ of targeted audience). The majority of participants completed the survey in day-1, with a decline in day-2 and day-3 (Figure 1). Demographics and related parameters (Table 1), showed that $64.1 \%$ and
$27.2 \%$ of the participants were aged 18 and 19 years respectively, while age range of participants was 17 to 21 . Female participants were much more compliant to take the survey, as $66.99 \%$ of participants were female.

Participants' handedness was predominantly right-handed $(88.35 \%)$. The participants' handedness was similar to parents' handedness in $78.6 \%$, while the participants' handedness was similar to siblings' handedness in $68.9 \%$. The number of spoken languages (Figure 3) was in the range of 1 to 5 , while the majority spoke either two languages $(35.9 \%)$ or three languages $(46.6 \%)$. Only one participant $(0.97 \%)$ spoke five languages.

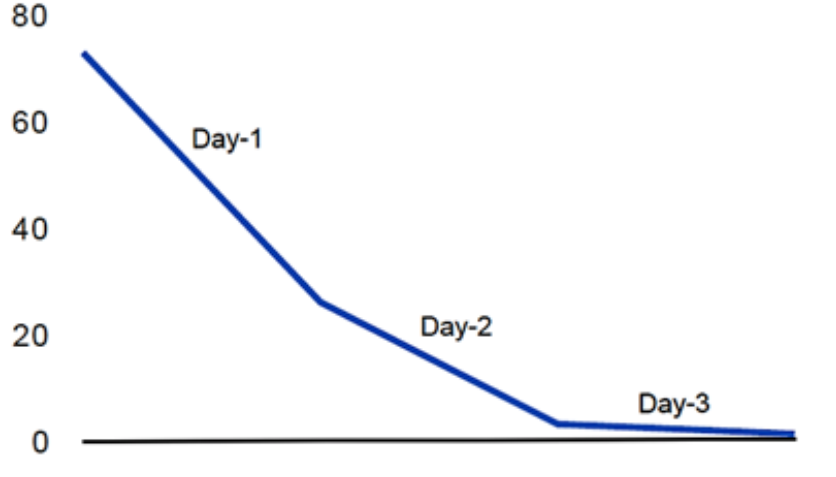

Figure 1: Survey's response-time curve
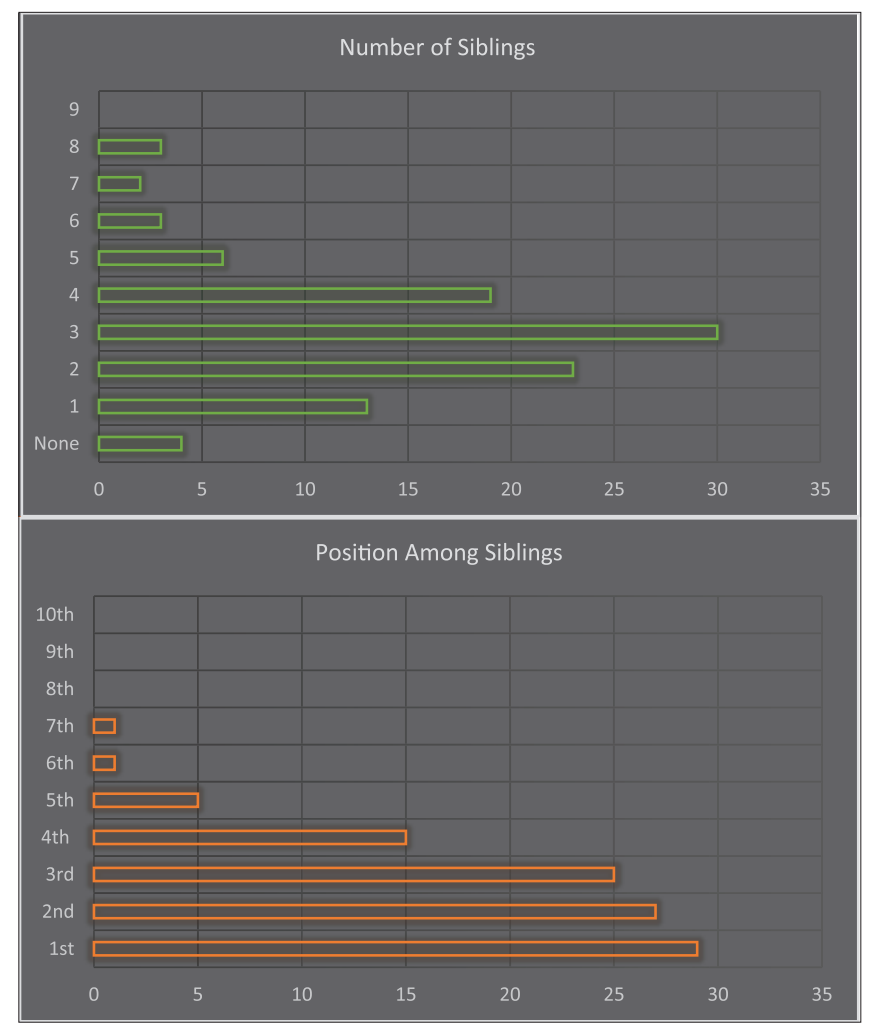

Figure 2: Demographic data related to survey participants' siblings 


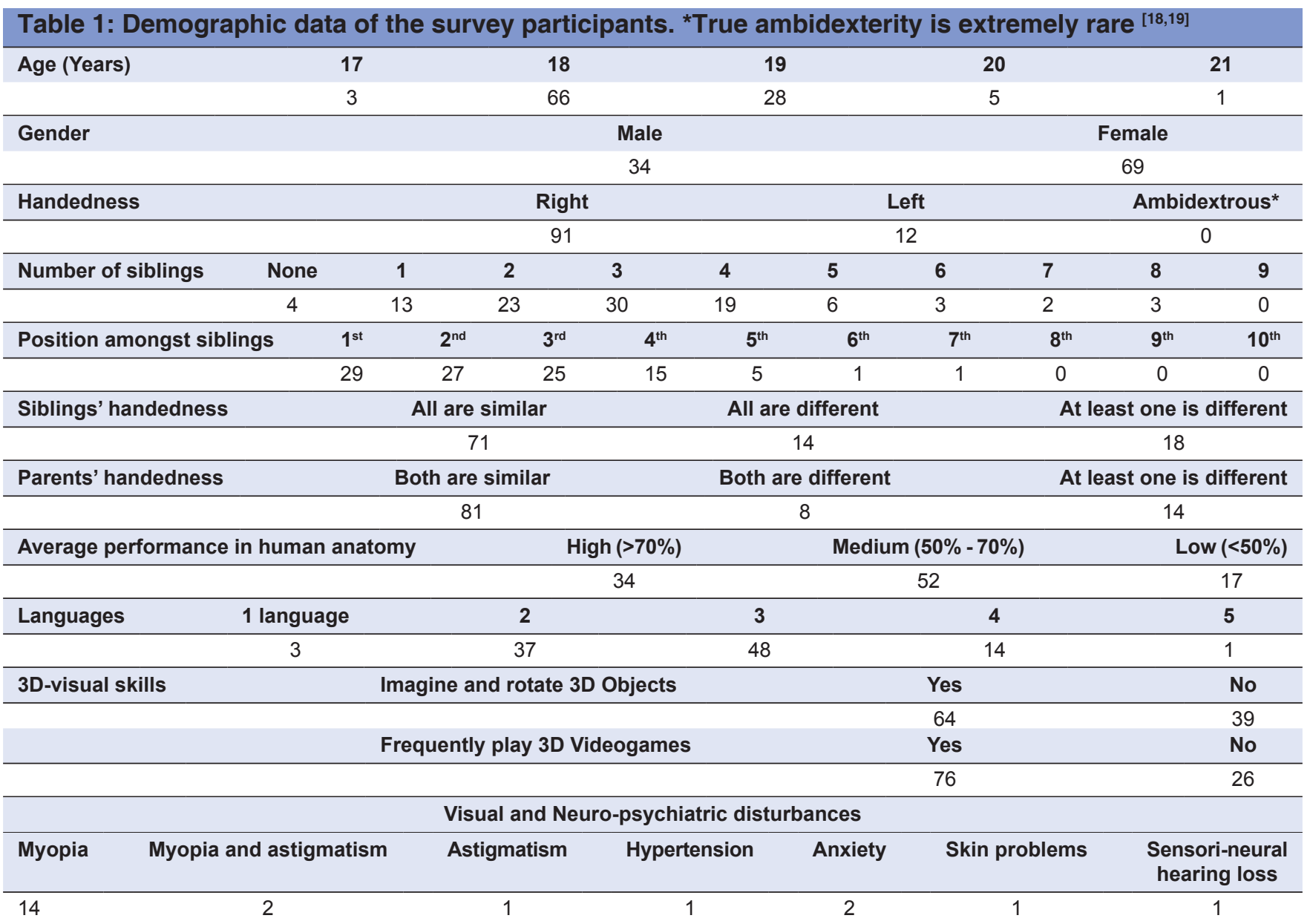

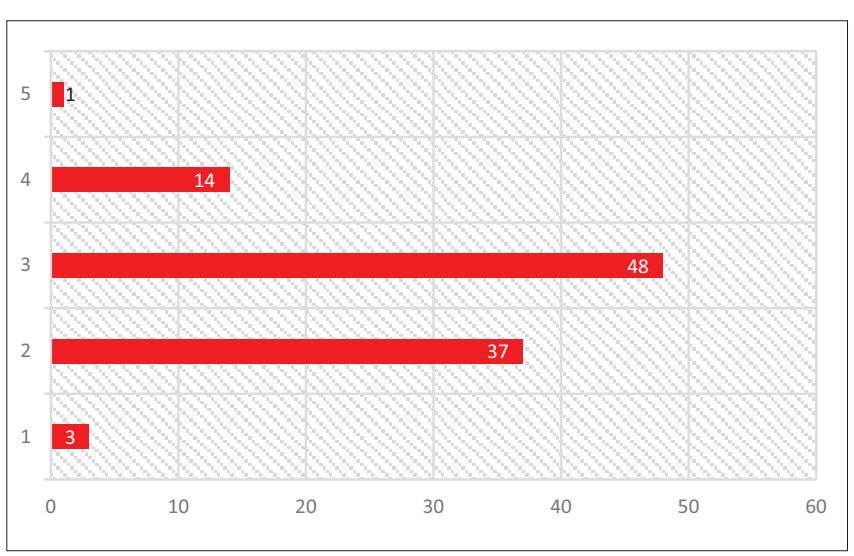

Figure 3: Number of spoken-written languages, maximum number of spoken languages was five

The three-dimensional visual imagination (visual analytic skills), were estimated in the survey by asking about: selfassessment of the ability to imagine and rotate $3 \mathrm{D}$-figures in their mind, and their frequent involvement in playing $3 \mathrm{D}$ videogames. This was further consolidated by estimating the visual intellectual quotient (IQ) of those who acclaimed good/high3D-visual analysis skills, via a professional IQ test. ${ }^{16}$ Those who failed to pass this IQ test were considered of an inferior 3D visual skills, and were labelled as "No" and subsequently dropped out from relevant statistical calculations. Participants with strong visual skills, based on subjective and objective evaluation, accounted for $64.1 \%$ of participants. In relation to this, those who had distinctionlevel (more than 70\%) of cumulative performance in Human Anatomy, accounted for $33 \%$, while $50.4 \%$ of participants had a percentile score of performance in the range of $50-70 \%$.

To make sure, that survey participants were free of illnesses that may interfere or lead to biases in this research, each participant was asked about medical problems including: visual, neurologic, psychiatric problems, and others. It has been found that, $16.5 \%$ had visual problems (including myopia and/or astigmatism), while $4.9 \%$ had other disorders (anxiety, hypertension, hyperhidrosis, sensorineural hearing loss, and skin problems). However, all these were evaluated and subsequently considered as non-limiting factors in taking this survey.

Concerning data on sectional MRI images, for sections-1, $66 \%$ visualized the section from above (a superior view), 
for section-2, $60.2 \%$ visualized the section from above (superior), for section- $3,47.6 \%$ visualized the section from above (superior), and for section- $4,51.5 \%$ visualized the section from above (superior). Obviously, the rest of the participants, visualized these four sections from below (an inferior view).

The application of Chi-square and Pearson's correlation test (Tables 2 and 3), revealed that there was no any statistical correlation between the visual analytic skills and any of the tested representative parameters of cerebral dominance. These parameters included: handedness, gender, and number of spoken-written languages (Tables 2 and 3). Significance was considered at a p-value of 0.05 or less. In relation to subject's handedness the $p$-values were: 0.213 , $0.830,0.427,0.612$ for sections $1-4$ respectively while using Chi-square test, and $0.216,0.932,0.432,0.616$ for sections 1-4 respectively while using Pearson's correlation test.

This study can be further developed in subsequent designs in the future, to correlate the visual analytic skills versus cognitive enhancers, psychostimulants, and novel psychoactive substances. ${ }^{20,21}$

\section{CONCLUSION}

In this sample of Iraqi undergraduate medical students, who are considered as non-professional Anatomists. It was hypothesized prior to carrying out this survey that there was a positive correlation between visual analytic skills and certain predefined parameters. These parameters are neurologic manifestations of cerebral dominancy, particularly handedness, gender, and the number of spoken languages. In other words, and in relation to subject's handedness, left-handed participants could potentially visualize objects, including MRI

\begin{tabular}{|c|c|c|c|c|}
\hline & $\begin{array}{c}\text { Section } \\
\text { no. } 1\end{array}$ & $\begin{array}{c}\text { Section } \\
\text { no. } 2\end{array}$ & $\begin{array}{c}\text { Section } \\
\text { no. } 3\end{array}$ & $\begin{array}{c}\text { Section } \\
\text { no. } 4\end{array}$ \\
\hline Gender & 0.259 & 0.930 & 0.362 & 0.531 \\
\hline Language & 0.682 & 0.530 & 0.246 & 0.641 \\
\hline Handedness & 0.213 & 0.830 & 0.427 & 0.612 \\
\hline 3D & 0.853 & 0.876 & 0.565 & 0.693 \\
\hline
\end{tabular}

\begin{tabular}{|c|c|c|c|c|}
\hline & $\begin{array}{c}\text { Section } \\
\text { no. } 1\end{array}$ & $\begin{array}{c}\text { Section } \\
\text { no. } 2\end{array}$ & $\begin{array}{c}\text { Section } \\
\text { no. } 3\end{array}$ & $\begin{array}{c}\text { Section } \\
\text { no. } 4\end{array}$ \\
\hline Gender & 0.263 & 0.931 & 0.366 & 0.535 \\
\hline Language & 0.267 & 0.849 & 0.209 & 0.318 \\
\hline Handedness & 0.216 & 0.932 & 0.432 & 0.616 \\
\hline 3D & 0.855 & 0.878 & 0.670 & 0.696 \\
\hline
\end{tabular}

images used in this study, differently from right-handed participants.

However, this cross-sectional analysis, which was of a randomized and a single-blinded design, revealed a confirmatory statistical evidence, the absence of any form of correlation and the wrongfulness of the pre-studied assumption of the presence of correlation between parametric manifestations of cerebral lateralization and complex visual analytics skills.

\section{LIMITATIONS}

Limitations of this study can be summarized in

1. Number of participants is relatively small, 103 participants accounting for $34.68 \%$ of the targeted audience.

2. Only $1^{\text {st }}$ year (year-1) undergraduate medical students were included in the survey, while excluding year-2. The exclusion was based on the fact that year-2 students finished the lower limb module of Human Anatomy a year ago. Therefore, there Anatomical knowledge was not fresh enough, as compared with year-1.

3. Cross sectional MRI images were only considered, while excluding coronal and sagittal MRI sectional images. These types of sections may require more experienced and post-graduate level of students of Human Anatomy.

4. Sectional MRI images, all belonged to the lower limb, while excluding other regional Anatomy images. Again, using more than one region of Anatomy, required a more advanced students of Human Anatomy.

5. Only four sectional anatomy images were used in the survey. Initially, the survey included 10 sectional MRIs, but it was subsequently lowered to six and then to four, due to lack of compliance (interest in taking the survey) in an initial pilot design of the survey. The survey evidently requires hightime-commitment to complete, for which many medical student lack.

\section{ACKNOWLDGMENTS}

Our gratitude and regards to Professor Dr. Ali Al-Shalchy for his orientation concerning the ethical approval acquisition of this study. Professor Al-Shalchy is a neurosurgeon, and a member of the royal academy of surgeons, and he is current dean of the faculty of Medicine at the University of Baghdad. Appreciation and gratitude to all of the $1^{\text {st }}$-year undergraduate medical students, who participated in this survey, and best of wishes to them in their future endeavors, and as future doctors of humanity and in our beloved country Iraq. 


\section{REFERENCES}

1. Wood IK. Neuroscience: Exploring the brain. Journal of Child and Family Studies. 1996;5(3):377-379.

2. Tortora GJ, Derrickson BH. Principles of anatomy and physiology. John Wiley \& Sons; 2008 Apr 11.

3. White LE, Lucas G, Richards A andPurves D. Cerebral asymmetry and handedness. Nature 1994 Mar.

4. Shlain L. Leonardo's Brain: Understanding Da Vinci's Creative Genius. Rowman \& Littlefield; 2014 Oct 21.

5. Baum N. Thinking Like Leonardo da Vinci and Its Implications for the Modern Doctor. The Journal of medical practice management: MPM 2013; 29(2):137.

6. Weiss PH, Ubben SD, Kaesberg S, Kalbe E, Kessler J, Liebig T, et al. Where language meets meaningful action: a combined behavior and lesion analysis of aphasia and apraxia. Brain Structure and Function 2016;221(1):563-576.

7. Boughner J, Rolian C, editors. Developmental Approaches to Human Evolution. John Wiley \& Sons; 2016 Jan 22.

8. Carter $\mathrm{R}$ and Frith $\mathrm{CD}$. Mapping the mind. Univ of California Press; 1998.

9. Riès SK, Dronkers NF and Knight RT. Choosing words: left hemisphere, right hemisphere, or both? Perspective on the lateralization of word retrieval. Annals of the New York Academy of Sciences 2016; Jan 1.

10. Boughner J, Rolian C, editors. Developmental Approaches to Human Evolution. John Wiley \& Sons; 2016 Jan 22.

11. Dehaene S, Spelke E, Pinel P, Stanescu R and Tsivkin S. Sources of mathematical thinking: Behavioral and brain-imaging evidence. Science 1999; 284(5416):970-974.
12. Google. Google Forms. https://www.google.com/forms/about/ (accessed 20 April 2016).

13. Google. Google Forms. https://docs.google.com/forms/ d/1dq1vE-AEDAV5WOnlOOz7mFIrrPLt7WYQWjpLRV5VRxU/ closedform (accessed 20 April 2016).

14. Singh H, Parvez S. Atlas of Human Anatomy on MRI: Spine Extremities Joints, $1^{\text {st }}$ ed. India: Jaypee Brothers Medical Pub; 2012.

15. University of Baghdad. Iraqi Network Learning Environment. http://inle.education/(accessed 20 April 2016).

16. The International High IQ Society. About the International High IQ Society. https://www.ihiqs.org/pages/about (accessed 20 April 2016).

17. Interactivate. Statistical Tools. http://www.shodor.org/ interactivate/activities/(accessed 23 April 2016).

18. Oldfield RC. The assessment and analysis of handedness: the Edinburgh inventory. Neuropsychologia. 1971 Mar 31;9(1):97-113.

19. Fagard J, Chapelain A and Bonnet P. How should "ambidexterity" be estimated?. Laterality: Asymmetries of Body, Brain and Cognition 2015; 20(5):543-570.

20. Al-Imam A, Santacroce R, Roman-Urrestarazu A, Chilcott R, Martinotti G, Bersani G, et al. Captagon: Use and Trade in the Middle East. Human Psychopharmacology: Clinical and Experimental $2016 \mathrm{http} / / /$ researchprofiles.herts.ac.uk/ portal/en/publications/captagon-use-and-trade-in-the-middleeast(e84aeb59-46c2-4bc2-bea6-09aadd1bfe9c).html.

21. Lutfi AM. A quick analysis in relation to the electronic commerce of performance-and image-enhancing drugs based on the infrastructure of the world wide web. Asian Journal of Medical Sciences 2016;7(4):88-93.

\section{Authors Contribution:}

NA - Critical analysis, Editing the manuscript, Research group and co-authors' coordination, Statistical analysis; AA - Study design, Survey design, Literature review, Statistical Analysis; MI - Study design, Survey design, Survey distribution, Proofreading the manuscript; MK - Survey design, Survey distribution, Critical analysis; SA - Survey design, Survey distribution, Critical analysis.

Source of Support: Nil, Conflict of Interest: None declared. 